\title{
Regulating double-stranded DNA break repair towards crossover or non-crossover during mammalian meiosis
}

\author{
Frédéric Baudat \& Bernard de Massy* \\ Institute of Human Genetics, UPR1142/CNRS, 141 rue de la Cardonille, 34396 Montpellier cedex 5, France; \\ Tel: +33-049-9619972; Fax: +33-049-9619901; E-mail: bdemassy@igh.cnrs.fr \\ * Correspondence
}

Key words: crossover, DSB repair, meiosis, non-crossover, recombination

\begin{abstract}
During meiosis the programmed induction of DNA double-stranded breaks (DSB) leads to crossover (CO) and non-crossover products (NCO). One key role of $\mathrm{CO}$ is to connect homologs before metaphase I and thus to ensure the proper reductional segregation. This role implies an accurate regulation of $\mathrm{CO}$ frequency with the establishment of at least one $\mathrm{CO}$ per chromosome arm. Current major challenges are to understand how $\mathrm{CO}$ and $\mathrm{NCO}$ formation are regulated and what is the role of $\mathrm{NCO}$. We present here the current knowledge about $\mathrm{CO}$ and $\mathrm{NCO}$ and their regulation in mammals. CO density varies widely along chromosomes and their distribution is not random as they are subject to positive interference. As documented in the mouse and human, a significant excess of DSB are generated relative to the number of CO. In fact, evidence has been obtained for the formation of NCO products, for regulation of the choice of DSB repair towards CO or NCO and for a CO specific pathway. We discuss the roles of Msh4, Msh5 and Sycp1 which affect DSB repair and probably not only the CO pathway. We suggest that, in mammals, the regulation of NCO differs from that described in Saccharomyces cerevisiae.
\end{abstract}

\section{Introduction}

In most sexually reproducing eukaryotes, at the beginning of the first meiotic prophase, a unique set of events is programmed: the induction of DNA double-stranded breaks (DSB) at various locations in the genome of each meiotic cell. These DSB are generated by the catalytic activity of Spo11 in a reaction, in part, similar to that described for type II DNA topoisomerases (Keeney 2001). The repair of these DSB by recombination with a chromatid from the homologous chromosome generates two types of recombinant molecules, reciprocal exchanges or crossover (CO) and gene conversion without crossover (NCO). CO plays an essential mechanical role for the reductional division at meiosis I by connecting homologs and thus allowing their proper orientation at metaphase I (Petronczki et al. 2003). Thus, at least one $\mathrm{CO}$ per chromosomal arm is required for the proper reductional division.

Studies in Saccharomyces cerevisiae have shown that $\mathrm{CO}$ and $\mathrm{NCO}$ are the products of two distinct DSB repair pathways (Allers \& Lichten 2001, Hunter \& Kleckner 2001) (Figure 1): after DSB formation and ends processing, a putative intermediate common to both $\mathrm{CO}$ and NCO pathways, and resulting from the strand exchange reaction of one DSB end, is formed. NCO products are thought to be generated by a mechanism called synthesis-dependent strand annealing (SDSA) involving the extension of the 3' invading end by a DNA polymerase, followed by its displacement and re-annealing onto the other end of the broken chromatid. The initiating chromatid thus contains heteroduplex DNA, which can lead to gene 


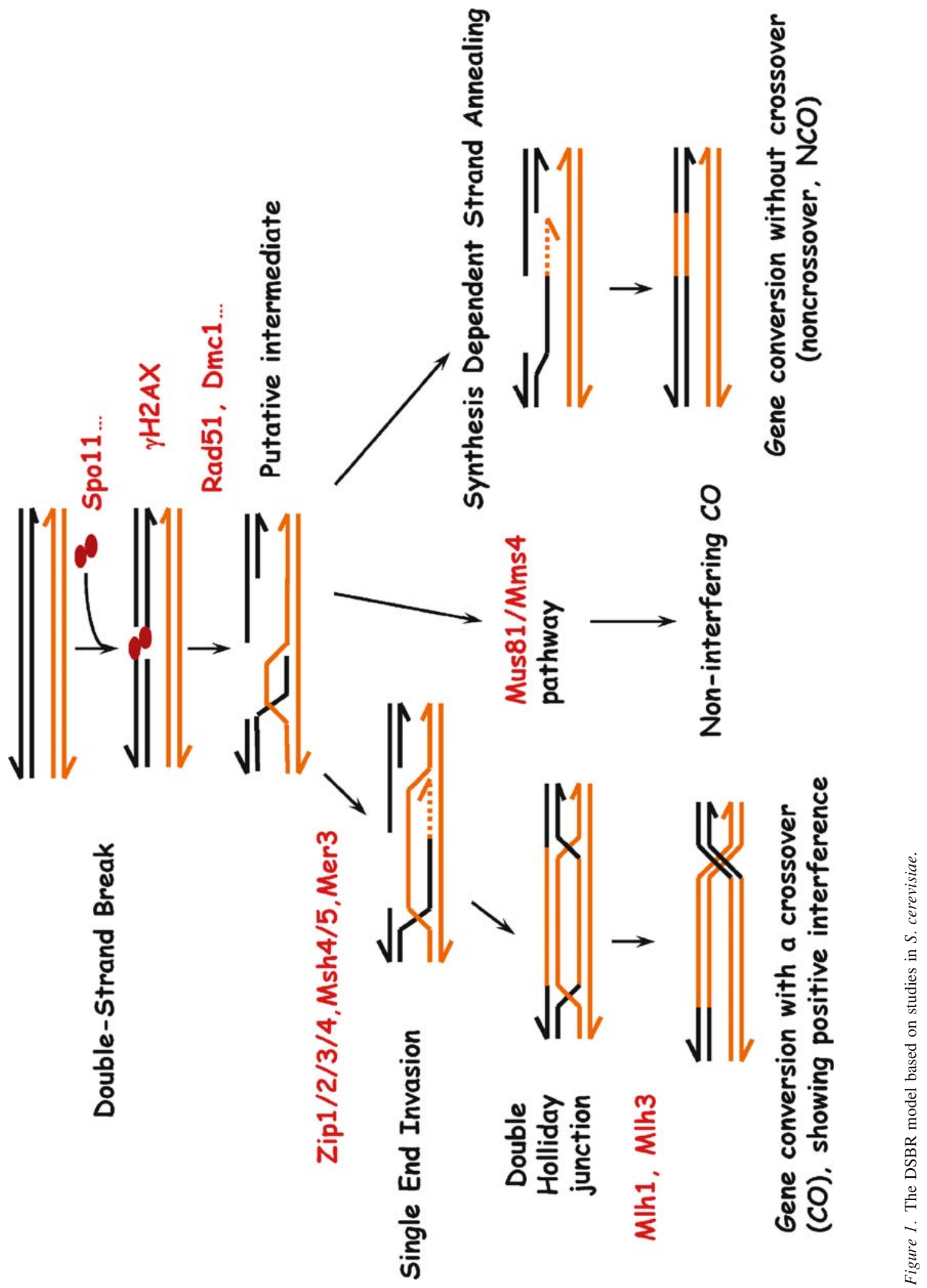


conversion without crossover upon mismatch repair. Mutants specifically altering this pathway have not been identified so far. It can be considered as a default pathway and its function is discussed below. Along the CO pathway, a single-end invasion intermediate (SEI) has been identified and is converted into a double Holliday junction by the annealing of the free DSB end to the displaced strand. This double Holliday junction is resolved such as to generate an exchange of flanking markers, and upon mismatch repair of heteroduplex DNA, a CO product with gene conversion is formed. Several functions have been shown to be specifically required for the $\mathrm{CO}$ pathway. These are called the ZMM functions, that include ZIP1, ZIP2, ZIP3, ZIP4, MSH4, MSH5 and MER3 genes (ZMM) (Borner et al. 2004). These functions seem to act at the time or soon after DSB formation at the leptotene/zygotene stage of meiotic prophase and one of their roles is to antagonize the anti-crossover activity of the Sgs1 helicase (Jessop et al. 2006). Strains carrying mutations in one of these genes have a reduction of $\mathrm{CO}$ whereas $\mathrm{NCO}$ are, in general, not affected. In addition, formation of $\mathrm{CO}$ in this pathway also requires Mlh1 and Mlh3 proteins, thought to act at a late step in the recombination reaction (Hunter \& Borts 1997, Wang et al. 1999, Alpi et al. 2003, Argueso et al. 2004). A unique manifestation of $\mathrm{CO}$ regulation is positive interference which leads to the non-random distribution of $\mathrm{CO}$ that are more widely spaced than expected (Jones 1984). In ZMM mutants the remaining CO do not show interference as measured genetically, and most depend on Mus81/Mms4 (De Los Santos et al. 2003). Interestingly, in $S$. pombe, where CO do not show interference, $\mathrm{CO}$ are completely dependent on Mus81/Eme1 (the S. pombe homolog of Mus81/ Mms4) and go through a single Holliday junction intermediate (Hollingsworth \& Brill 2004, Cromie et al. 2006).

One current major issue is to understand how the decision for $\mathrm{CO} / \mathrm{NCO}$ is made (Borner et al. 2004, Bishop \& Zickler 2004) and how the proper number and position of $\mathrm{CO}$ are achieved in each meiotic cell. Understanding this obviously requires an understanding of the whole pathway of DSB repair and to detect and analyze not only $\mathrm{CO}$ but also those DSB repair events not leading to $\mathrm{CO}$ in order to answer the following questions: When is a DSB event committed to be repaired as a CO? How is the non-random spacing of $\mathrm{CO}$ regulated? Why are there recombination events in excess of the number of $\mathrm{CO}$ and what are their roles?

Here, we present the current information about meiotic DSB repair mechanisms and regulation in mammals related to these issues of CO control. Most of the data presented takes advantage of recent studies in humans and mice. We first outline the evidence for DSB formation and repair and describe the data showing the excess of DSB repair events as compared to $\mathrm{CO}$. We then summarize the large amount of information about $\mathrm{CO}$ distribution and frequencies as well as the limited information about $\mathrm{NCO}$ and the evidence for $\mathrm{NCO} / \mathrm{CO}$ control. We then discuss the results obtained from the analysis of mice carrying mutations in ZMM orthologs (Msh4, Msh5 and Sycp1) and suggest interpretations for their roles along the DSB repair pathway.

\section{Conservation of the DSB repair pathway}

\section{Initiation by Spol1-generated DSB}

The Spo11 protein is widely conserved and the current evidence for initiation of meiotic recombination by DSB in mammals comes from the observation that a high level of $\mathrm{H} 2 \mathrm{AX}$ phosphorylation is detected at the leptotene stage of meiotic prophase. In mice this phosphorylation has been shown to be Spo11-dependent (Mahadevaiah et al. 2001). In addition, the detection of Spo11-oligo molecules from wild-type mouse testis strongly supports the notion that Spo11 is catalytically active in meiosis (Neale et al. 2005). These Spo11-oligo covalent molecules, where Spo11 is bound to the $5^{\prime}$ end of a short oligonucleotide (15-30 bp long), are thought to result from the processing of Spo11-DNA cleavage complexes that have been released by an unknown endonuclease activity. DNA breaks have also been visualized in mouse testis sections by assays allowing the detection of ends with $3^{\prime}$ overhangs (Zenvirth et al. 2003), and at a mouse CO hotspot by the use of terminal transferase (Qin et al. 2004). One property of initiation by DSB and repair as depicted in Figure 1 is that the initiating chromosome is the recipient of information. This prediction of the model has indeed been confirmed at several CO hotspots in human and mouse (Jeffreys \& Neumann 2002, Yauk et al. 2003, Jeffreys \& Neumann 2005: Baudat and de Massy, PLoS Genetics, in press). 
Given that the phosphorylation of H2AX at leptotene spreads over large overlapping chromatin domains the total number and distribution of DSB could not be determined.

\section{$D S B$ repair events: an excess compared to $C O$}

Given the model outlined in Figure 1, one predicted intermediate common between $\mathrm{CO}$ and $\mathrm{NCO}$ is a strand exchange product. The two major strand exchange proteins acting in meiosis, Rad51 and Dmc1 (Shinohara 2004), have been analyzed by immunocytochemistry. In mouse and human spermatocytes and oocytes, Rad51 and Dmc1 co-localize and form multiple foci at leptotene and zygotene (Ashley et al. 1995, Plug et al. 1996, Barlow et al. 1997, Moens et al. 1997). Rad51 foci are detected in early leptotene and appear to be mostly located on the chromosome axis. The number of foci decreases as synapsis proceeds from zygotene to early pachytene and goes down to an undetectable level at the middle of pachytene (Moens et al. 2002, OliverBonet et al. 2005). Thus, the number of Rad51/Dmc1 foci peaks at leptotene to about 200-400 in mice and humans, with differences between sexes (Table 1). These values are a minimum estimate of the total number of DSB repair events, which exact value is therefore unknown. In any case the number of these foci by far exceeds the number of $\mathrm{CO}$ (Table 1 and see below, 'Properties of CO products'), raising the questions of why are so many DSB generated and how are their repair regulated? The decrease of Rad51/Dmc1 foci from leptotene to the middle of pachytene is thought to reflect the progression of the

Table 1. Quantitative data on meiotic recombination events in humans and mice (male and female)

\begin{tabular}{|c|c|c|c|c|c|c|}
\hline & \multicolumn{3}{|l|}{ Mus musculus } & \multicolumn{3}{|l|}{ Human } \\
\hline & Female & Male & Reference & Female & Male & Reference \\
\hline \multirow[t]{5}{*}{ Rad51 foci } & $250-350$ & $230-270$ & Plug et al. 1996 & $350-400$ & & Lenzi et al. 2004 \\
\hline & $420-370(394)$ & & Kolas 2005 & & $91-262(152)$ & Barlow et al. 1997 \\
\hline & & $250-300$ & Tarsounas 1999 & & $104 \pm 28$ & Oliver-Bonet \\
\hline & & 300 & Moens et al. 2002 & & & \\
\hline & & 400 & de Vries et al. 2005 & & & \\
\hline \multirow[t]{8}{*}{ Mlh1 foci } & $23-33(28)$ & & Kolas 2005 & $48-102(70)$ & & Tease 2002 \\
\hline & $19-34(26.1)$ & & Lynn et al. 2005 & $10-107(60)$ & & Lenzi et al. 2004 \\
\hline & $26.6 \pm 3.2$ & & Koehler et al. 2006 & & $41-59(50.9)^{\mathrm{c}}$ & Barlow et al. 1998 \\
\hline & $24.1 \pm 2.6^{\mathrm{b}}$ & & Koehler et al. 2006 & & $49.1 \pm 4.8$ & Lynn 2002 \\
\hline & & 22.7 & Anderson et al. 1999 & & 50 & $\begin{array}{l}\text { Oliver-Bonet } \\
\text { et al. } 2005\end{array}$ \\
\hline & & 23 & Moens et al. 2002 & & $32-63(47.8)$ & $\begin{array}{l}\text { Codina-Pascual } \\
\text { et al. } 2006\end{array}$ \\
\hline & & $22.9^{c}$ & Froenicke 2002 & & & \\
\hline & & $21.5-24.3^{\mathrm{c}}$ & Koehler et al. 2002 & & & \\
\hline \multirow[t]{2}{*}{ Chiasmata } & & $22.6-23.9$ & $\begin{array}{l}\text { ref. in Anderson } \\
\text { et al. } 1999\end{array}$ & & $49.6-53.7$ & $\begin{array}{l}\text { ref. in Vallente } \\
\text { et al. } 2006\end{array}$ \\
\hline & 24.1 & & Woods et al. 1999 & & & \\
\hline \multirow[t]{3}{*}{ Genetic map (cM) } & $1385^{\mathrm{b}}$ & & Dietrich et al. 1996 & $\begin{array}{c}3799-4600 \\
(4400)\end{array}$ & $\begin{array}{c}2590-2813 \\
(2700)\end{array}$ & $\begin{array}{l}\text { ref. in Vallente } \\
\text { et al. } 2006^{\mathrm{d}}\end{array}$ \\
\hline & $1398^{\mathrm{b}}$ & & Rhodes 1998 & & & \\
\hline & 1817 & 1386 & Shifman et al. 2006 & & & \\
\hline 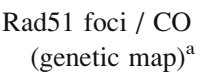 & $394 / 36=11$ & $250 / 28=9$ & & $370 / 88=4$ & $152 / 54=3$ & \\
\hline
\end{tabular}

${ }^{\mathrm{a}} 50 \mathrm{cM}$ correspond to an average of one $\mathrm{CO}$ per meiocyte.

${ }^{\mathrm{b}}$ M. musculus $\times$ M. spretus interspecific cross.

${ }^{\mathrm{c}}$ Autosomal Mlh1 foci only have been counted. One focus has to be added for taking into account the obligatory CO on the pseudo-autosomal region of the XY pair.

${ }^{\mathrm{d}}$ for additional data on human genetic maps, see also Lynn et al. 2004. 
repair events with the elimination of $\mathrm{Rad} 51 / \mathrm{Dmc} 1$ once the strand exchange reactions are complete. Several other proteins have been immunolocalized and found to mark recombination foci. These have been reviewed (Cohen \& Pollard 2001, Vallente et al. 2006) and will not be described here except for those whose analysis provides insight into the understanding of $\mathrm{CO} / \mathrm{NCO}$ pathways and $\mathrm{CO}$ regulation (see below, 'The $\mathrm{CO} / \mathrm{NCO}$ decision, the ZMM proteins in mammals').

\section{Properties of $\mathrm{CO}$ products}

\section{Frequency and distribution}

CO can be measured over the whole genome by cytological, genetic or population analysis. The total number of chiasma is around 23 in mice and 52 in humans, with differences between sexes (see below, 'Variations of CO activity'; Table 1). CO can also be monitored by the immunolocalization of the Mlh1 protein, whose foci have been shown to correspond to sites of chiasma (Marcon \& Moens 2003). In principle, a perfect correlation between the numbers of Mlh1 foci and $\mathrm{CO}$ is not necessarily expected given the potential existence of a Mlh1-independent CO pathway, as shown in $S$. cerevisiae for instance. In fact, in mice, Mlh1 is required for the formation of most, but not all, $\mathrm{CO}$ given the 10-20-fold reduction of $\mathrm{CO}$ frequencies in Mlh1-/- mice, which have therefore a residual level of $\mathrm{CO}$ activity corresponding to 5-10\% of wild-type (Woods et al. 1999, Guillon et al. 2005). We note a slight excess of $\mathrm{CO}$ as measured genetically as compared to Mlh1 foci in wild-type mice, which could be due to technical bias or to the presence of a small proportion of Mlh1-independent CO in wild-type mice (Table 1). These cytological analyses also show that at least one $\mathrm{CO}$ is formed per chromosome arm both in humans and mice, apart from short heterochromatic arms from acrocentric chromosomes. This regulation of $\mathrm{CO}$ frequency is a manifestation of the rule of the obligatory CO. Interestingly, mouse strains carrying Robertsonian translocations have two CO per chromosome, one per euchromatic arm (Dumas \& BrittonDavidian 2002). The rule of the obligatory $\mathrm{CO}$ is also observed between the $\mathrm{X}$ and $\mathrm{Y}$, where one $\mathrm{CO}$ is always observed in the pseudoautosomal region. A second level of $\mathrm{CO}$ regulation is shown by the measures of distances between chiasmata or Mlh1 foci, or by genetic distances. These show that, both in mouse and human, $\mathrm{CO}$ are not randomly distributed and are more evenly spaced than expected if they occurred independently, a phenomenon defined as positive interference (Lawrie et al. 1995, Laurie \& Hulten 1985, Anderson et al. 1999, Broman \& Weber 2000, Broman et al. 2002).

The recent genetic maps determined by pedigree or based on population analysis provided a genomewide high-resolution map of $\mathrm{CO}$ distribution both in the human and the mouse genomes (McVean et al. 2004, Myers et al. 2005, Shifman et al. 2006). These approaches confirmed at large scale previously established properties of $\mathrm{CO}$ distribution and led to additional conclusions: $\mathrm{CO}$ are not randomly distributed, but occur in multiple specific regions of the genome called CO hotspots (de Massy 2003, Kauppi et al. 2004). A hotspot is a region $1-2 \mathrm{~kb}$ wide where $\mathrm{CO}$ are clustered, as a result of localized initiation events (see below). The average spacing between hotspots is $50-100 \mathrm{~kb}$ and hotspot activities vary over three to four orders of magnitude, from 0.9 to $0.0005 \mathrm{cM}$ as determined in the human genome. In the human genome the number of hotspots is estimated around 25 000-50 000 (Myers et al. 2005). The variations of hotspot density and activity along chromosomes result in domains with high (jungles) or low (desert) recombination activity. Most sub-telomeric regions are recombination jungles in male meiosis whereas centromeric regions are recombination deserts. However, the determinants of $\mathrm{CO}$ variation along chromosomes are not known, even though some factors correlated with $\mathrm{CO}$ density are beginning to be analyzed (Buard \& de Massy 2007, TIG in revision).

In addition, molecular analysis of recombinant products has been performed in specific regions of the human and mouse genomes by allele-specific PCR that have allowed not only measures of $\mathrm{CO}$ frequencies, but also the determination of the distribution of exchange points at several $\mathrm{CO}$ hotspots. In all cases, 17 human hotspots (Holloway et al. 2006) and two mouse hotspots (Guillon \& de Massy 2002, Yauk et al. 2003) exchange points follow a bell-shaped curved with a peak of density in a narrow interval (on the order of a few hundred bp) predicted to correspond to the region of initiation (Figure 2). This distribution of exchange points is coherent with the DSB repair model where 

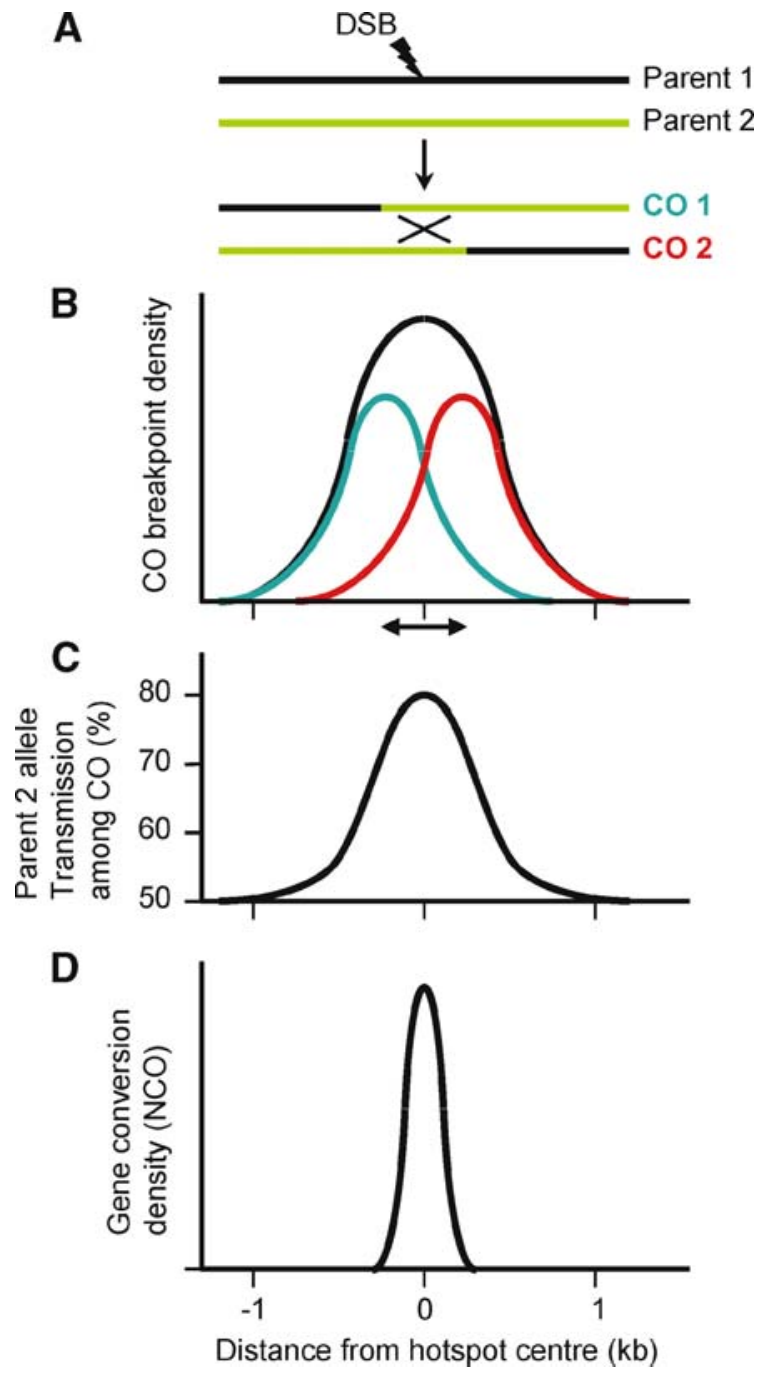

Figure 2. Theoretical distributions of $\mathrm{CO}$ breakpoints and gene conversions resulting from NCO events at a hotspot. A: Schematic representation of the chromatids involved in one $\mathrm{CO}$ event at a hotspot. The Spo11-generated DSB occurs only on Parent 1 molecule. $\mathrm{CO} 1$ and $\mathrm{CO} 2$ represent both exchange products generated by one reciprocal CO event. B: Distribution of the CO breakpoints along the hotspot. DSB are highly localized at the hotspot centre. In blue, $\mathrm{CO} 1$ breakpoints. In red, $\mathrm{CO} 2$ breakpoint; these two curves overlap due to the spreading of DSB sites. In black, $\mathrm{CO} 1+\mathrm{CO} 2$ exchange points. The double arrow represents the average length of gene conversion tracts associated with $\mathrm{CO}$. C: Percentage of transmission of Parent 2 alleles (from the noninitiating chromosome) into CO products. D: Density of gene conversion resulting from $\mathrm{NCO}$ events.

intermediates extend to a variable distance from initiation (Figure 1). The analysis of the distribution of $\mathrm{CO}$ exchange points can also provide information on the initiation activity of each homolog, and on the average size of gene conversion tracts involved in the $\mathrm{CO}$ events. Indeed, when initiation occurs on one chromatid, because mismatch repair is directed and leads to a conversion bias towards the unbroken chromatid, the exchange points of each $\mathrm{CO}$ product are distributed asymmetrically on either side of the region of initiation. In a population of $\mathrm{CO}$ products a differential initiation activity on each homolog results in a transmission distortion for markers located in the region of initiation and correlated with a non-overlapping distribution of the two types of reciprocal products (Figure 2). When a strong distortion is observed, the distance between these two distributions provides an estimate of the average gene conversion tract. Several such cases have been observed in humans and mice, and have shown that conversion tract lengths associated with $\mathrm{CO}$ are around 500 bp (Jeffreys \& Neumann 2002, Baudat and de Massy, PLoS Genetics, in press). An important output from direct molecular analysis is that they have allowed a real-time analysis of $\mathrm{CO}$ formation and showed at one CO hotspot in the mouse genome ( $P s m b 9)$ that $\mathrm{CO}$ products are formed at the end of the pachytene stage in male meiosis (Guillon \& de Massy 2002). Therefore, about 8 days separate DSB from CO formation in male meiosis.

\section{Variations in $\mathrm{CO}$ activity}

The overall number and distribution of $\mathrm{CO}$ are not identical for all individuals in a given species; on the contrary, they display both DNA sequence-dependent and -independent variations. The differences between genders also illustrate the occurrence of DNA sequence-independent variations: in humans and to a lesser extent in mouse, overall $\mathrm{CO}$ rates are significantly higher in females than in males (Table 1), with, however, higher male-specific densities of $\mathrm{CO}$ in the centromere-distal sub-telomeric regions (Broman et al. 1998, Kong et al. 2002, Shifman et al. 2006). The number and distribution of MLH1 foci in XY female mice are similar to those observed in normal $\mathrm{XX}$ females rather than those observed in normal XY males, demonstrating that the biological sex rather than the genotype (i.e. the XY chromosome pair) determines the gender-specific pattern of meiotic recombination in this organism (Lynn et al. 2005). 
The genetic background is also a major determinant of the variations in the number and distribution of CO. Indeed, the comparison between several inbred mouse lines revealed small but reproducible differences in the overall number of MLH1 foci (Koehler et al. 2002). In crosses between different lines, such as those used for genetic linkage analyses, the pattern of recombination might be affected by additional factors, including the level of polymorphism and a possible genetic interaction between the genomes of both parents. For example, the female genetic maps generated from interspecific Mus m. domesticus $\times$ M. spretus backcrosses are not longer than the sex-averaged maps generated from $M$. musculus intraspecific intercrosses, despite the fact that female genetic maps are usually longer than their male counterparts (Dietrich et al. 1996 and Table 1). A recent comparison between both parent strains (C57BL/6 and SPRET) and their F1 hybrid confirmed that the number of MLH1 foci is reduced significantly in the hybrid (Koehler et al. 2006). In humans, differences among individuals are well documented, suggesting that genetic factors affect the overall CO rate (Broman et al. 1998, Kong et al. 2002, Lynn et al. 2004, Codina-Pascual et al. 2006). Besides these large-scale differences in $\mathrm{CO}$ number, the local distribution of $\mathrm{CO}$ can also depend on the DNA sequence. This is illustrated by the fact that several $\mathrm{CO}$ hotspots identified in the mouse MHC are specific for one or a few haplotypes (Shiroishi et al. 1995). Similarly, at several human CO hotspots, the $\mathrm{CO}$ rate was found to vary widely from one individual to another (Neumann \& Jeffreys 2006). In some cases, these variations were found to correlate with specific sequence polymorphisms located close to the center of the hotspot, suggesting that these variations of $\mathrm{CO}$ activity reflect variations in the rate of DSB formation (Jeffreys \& Neumann 2005).

\section{Properties of NCO products}

The large excess in the number of Rad51/Dmc1 foci over $\mathrm{CO}$ in mice and humans suggests that $\mathrm{NCO}$ outnumber $\mathrm{CO}$ in mammals (see above; Table 1). However, given that NCO events lead to a highly localized modification of the genotype, their detection requires specific tools and their description and analysis are still poorly documented in mammalian genomes. Most of the data comes from molecular analysis at a few specific $\mathrm{CO}$ hotspots in the human and mouse genomes. These studies have shown that most CO hotspots are also NCO hotspots, indicating that the same initiating regions can lead to both $\mathrm{CO}$ and NCO products (Guillon \& de Massy 2002, Jeffreys \& May 2004).

The relative ratio of $\mathrm{CO}$ to $\mathrm{NCO}$ was found to vary considerably from one hotspot to another, as shown by the comparison of several hotspots in the human genome. The measured values went from 1:2.7 to 4:1, and even to more than 12:1 at one hotspot where no NCO was detected (Holloway et al. 2006). It should be kept in mind that most NCO rates are probably under-evaluated in proportions which are difficult to estimate, given that their detection depends on the presence of polymorphic markers. Nevertheless, it is interesting to compare these values for individual hotspots with the predictions made at the scale of the whole genome, based on cytological analysis as described above (Table 1). Given that a significant fraction of NCO may remain undetected, these ratios indicate that $\mathrm{NCO}$ indeed represent a substantial proportion of the meiotic DSB repair events in mammals.

Several aspects of NCO formation have been compared with $\mathrm{CO}$. NCO are clustered at the center of $\mathrm{CO}$ hotspots, with a sharp decrease in gene conversion frequencies on both sides, indicating that both $\mathrm{CO}$ and $\mathrm{NCO}$ are the products of initiation events taking place in the same region (Jeffreys \& May 2004, Guillon et al. 2005). NCO products are formed with a kinetics similar to those of $\mathrm{CO}$, from middle to late pachynema (Guillon et al. 2005).

However, detailed mapping analyses have shown an amazing and unexpected difference between $\mathrm{CO}$ and NCO: the size of the conversion tract. Whereas the size of gene conversion tracts among $\mathrm{CO}$ products was found to be around $500 \mathrm{bp}$ (see above), among NCO the conversion tracts are considerably shorter, with a mean length estimated between 50 and 300 bp (Jeffreys \& May 2004, Guillon et al. 2005). This is consistent with the fact that NCO and $\mathrm{CO}$ are outcomes of different DSB repair pathways, involving different recombination intermediates. In fact, Mlh1 and Mlh3 were found to be required for CO but not NCO formation (Guillon et al. 2005; Svetlanov, Baudat, Cohen \& de Massy, personal communication). 


\section{The $\mathrm{CO} / \mathrm{NCO}$ decision, the $\mathrm{ZMM}$ proteins in mammals}

Four mammalian homologs of the yeast ZMM proteins have been identified: Sycp1 (Sage et al. 1995, Meuwissen et al. 1997, de Vries et al. 2005), Msh4 (Paquis-Flucklinger et al. 1997, Kneitz et al. 2000), Msh5 (Bocher et al. 1999, Her et al. 1999, Edelmann et al. 1999) and Mer3 (also called HFM1, Entrez IDs: 330149 and 164045 for mouse and human, respectively). The Mer3 gene and protein have not been analyzed. Msh4 and Msh5 have been detected in testes and ovaries only, and do not seem to be expressed at significant levels in other tissues. Protein-protein interaction studies of mouse and human proteins expressed in cultured cells indicate that Msh4 and Msh5 dimerize via their carboxytermini (reviewed in Kolas \& Cohen 2004). Msh4 interacts with Rad51 and Dmc1 (Neyton et al. 2004) and with Mlh1 (Santucci-Darmanin et al. 2000). Immunolocalization shows that Msh4 and 5 co-localize and form foci in zygotene until late pachytene (Lenzi et al. 2004). The number of mouse Msh4 foci goes from 142 to 47 at mid-pachytene (Kneitz et al. 2000) where it co-localizes with Mlh1 (SantucciDarmanin et al. 2000). Therefore, unlike S. cerevisiae, in mammals the number of Msh4 foci exceeds the number of CO. Msh4 foci are evenly distributed, show a low level of interference and their distribution is clearly different from that of Mlh1, which shows a strong level of interference (de Boer \& Heyting 2006). Mutant Msh4 or Msh5 mice show a strong synapsis defect, but have high levels of Rad51 foci (tested in Mhs4-/- mice only), possibly indicating a defect in the processing of recombination intermediates and a failure to establish or maintain stable homologous interactions (de Vries et al. 1999, Kneitz et al. 2000). While mutant spermatocytes undergo apoptosis at the end of zygotene/beginning of pachytene, oocytes proceed until diakinesis, but are completely eliminated at 1 week of age. Although these phenotypes indicate an early role for Msh4 and 5 in the process of DSB repair, these proteins have been proposed to also play a role late in recombination, based on their co-localization with Mlh1 and on in-vitro assays (Snowden et al. 2004). The observation of a major synapsis defect in Msh4 or 5 mutants and of the number and distribution of Msh4/5 foci suggest that, differently from $S$. cerevisiae, the role of these proteins is not restricted to the formation of $\mathrm{CO}$ regulated by interference. We propose that, in mammals, Msh4 could be involved in both the formation of $\mathrm{CO}$ and $\mathrm{NCO}$ by acting before the separation of $\mathrm{NCO} / \mathrm{CO}$ pathways. In mammals, $\mathrm{NCO}$ are therefore predicted to be subject to a specific constraint as revealed by the low level of interference between Msh4 foci, whereas no interference could be detected genetically between NCO in $S$. cerevisiae (Malkova et al. 2004). The action of Msh4 both in mammals and yeast could in fact be functionally similar and linked to interference control but operating at different times, and therefore at different stages, along the DSB repair pathway in these organisms (de Boer \& Heyting 2006, Fig. 3).

Sycp1 is a meiosis-specific protein homologous to S. cerevisiae Zip1 based on weak sequence homology, and is a transverse filament protein, part of the central element of the synaptonemal complex (Schmekel et al. 1996). Sycp1 mutant male mice show defects in prophase progression and are defective in synaptonemal complex formation. However, pachytene spermatocytes have their homologs aligned at distance, suggesting that homologous interactions have been established but not converted into tight associations (de Vries et al. 2005). In Sycp1-/- mice, Rad51 and Msh4 form foci as in wild-type mice, showing that Sycp1 is not required for the low level of interference observed on Msh4 foci at late zygotene. However, these foci persist longer than in wild-type mice, suggesting a defect during the process of DSB repair. Indeed, $\gamma-\mathrm{H} 2 \mathrm{AX}$ foci also persist longer and Mlh1/3 foci do not form. Whether DSB are repaired toward NCO remains to be determined. Among the rare spermatocytes that reach metaphase I, most chromosomes form univalents suggesting a CO defect. (Whether Sycp1 is required for interference between Mlh1 foci in wildtype mice cannot be determined.) In yeast the Sycp1 homolog, Zip1, is not required for the establishment of interference between Zip2 foci which largely co-localize with Msh4 (Novak et al. 2001). This could be coherent with the normal level of interference between Msh4 foci in Sycp1-/- mice. Another property of the ZMM proteins in S. cerevisiae is that they define the SIC (Synapsis Initiation Complex). Several arguments have led to the proposition that SIC are located at the same sites where crossovers will form (Henderson \& Keeney 2005). In humans, analysis of synapsis initiation has indeed suggested a 
limited number of sites preferentially located near sub-telomeric regions in correlation with $\mathrm{CO}$ density (Brown et al. 2005).

\section{Unexplored DSB repair events}

We have mentioned above that the number of meiotic DSB far exceeds the final number of interhomolog $\mathrm{CO}$ (see Table 1). Although recent data show that a significant fraction of DSB are repaired leading to NCO between homologs (see above), alternative, unexplored possibilities have to be considered. Although meiotic recombination events are channeled towards homologous interactions, the fraction that possibly goes through interactions between sister chromatids is unknown. This control is in part mediated by meiotic specific cohesins. Indeed, a mutant defective for a meiosisspecific cohesin (Rec8) displays synapsis associating sister chromatids rather than synapsis between homologous chromosomes (Xu et al. 2005).

In addition, DSB repair can occur by intra-chromatid interactions. Meiotic intra-allelic rearrangements have been observed at unstable minisatellites (Buard \& Vergnaud 1994, Jeffreys et al. 1994). The localization of these tandem-repeat DNA sequences in $\mathrm{CO}$ hotspots strongly suggests that these rearrangements are a product of the repair of meiotic DSB (Jeffreys et al. 1998, Buard et al. 2000). Though rearranged products resulting from intra- or interchromatids cannot be distinguished from each other, some of them might result from intra-chromatid events. Since the inter-homolog $\mathrm{CO}$ are the sole

\section{S. cerevisiae}

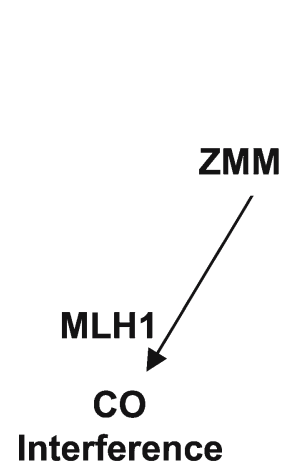

\section{DSB}

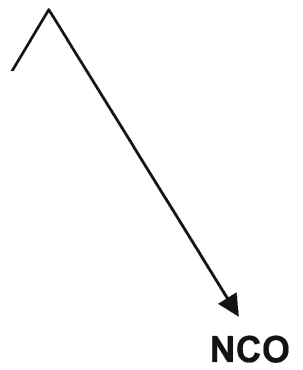

No Interference recombination products to have been counted exhaustively at the scale of the whole genome so far, the respective proportions of the other types of products (inter-homolog NCO, inter-sister recombination and intra-chromatid recombination) remain unknown.

An alternative DSB repair pathway to homologous recombination is non-homologous end joining (NHEJ), which results in the ligation of the ends on both sides of the DSB. This pathway is a major one for repairing DNA DSB in somatic cells, especially during the G1 phase of the cell cycle. During meiosis, KU70, a component essential to the NHEJ, is absent from the spermatocyte nuclei during the leptotene and zygotene stages of prophase, when meiotic DSB are generated and processed. Moreover, at the later pachytene stage, KU70 reappears but is mostly concentrated in the sex body, which corresponds to the chromatin domain of the nonpseudoautosomal regions of the $\mathrm{X}$ and $\mathrm{Y}$ chromosomes (Goedecke et al. 1999). These observations suggest that the NHEJ is indeed prevented during the course of meiotic recombination, at least in the autosomes.

During male meiosis the sex chromosomes do not have a homolog, with the exception of the short pseudoautosomal region. DSB are likely to be generated on the $\mathrm{X}$ and $\mathrm{Y}$ since RAD51 foci form on their axes (except for the heterochromatic region of the Y). However, these foci accumulate and persist until late pachytene, and are different from the autosomal foci that disappear progressively during the progression into the pachytene stage (see above). It is therefore possible that the DSB generated on the $\mathrm{X}$ and $\mathrm{Y}$ chromosomes are repaired by one of the

\section{Mammals}

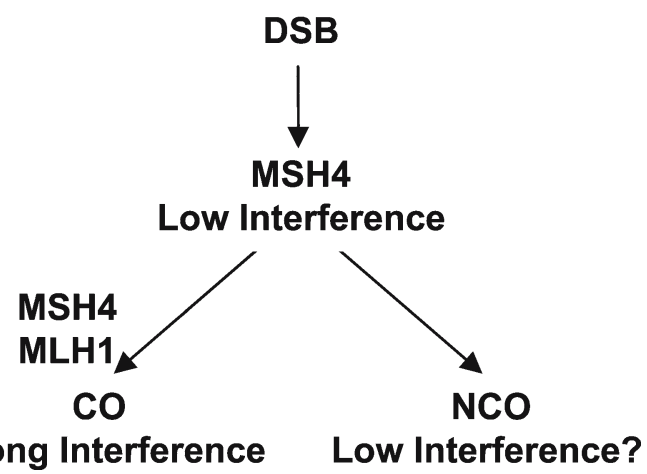

Figure 3. Regulations of $\mathrm{NCO} / \mathrm{CO}$ decision in S. cerevisiae and mammals. 
alternative pathways mentioned above. The persistence of the X/Y RAD51 foci through the pachytene stage suggests that this alternative repair might necessitate the relaxation of some constraints (for instance, against inter-sister recombination) that would be imposed at earlier stages to favor interhomolog recombination interactions.

\section{Conclusions}

The current data suggest that a large number of DSB are induced at the beginning of meiosis and lead to several hundred DSB repair events in mammals. These repair events are highly regulated to result in the formation of at least one $\mathrm{CO}$ per chromosome arm. These CO show a high level of interference and depend on Mlh1/3 proteins, which co-localize with $\mathrm{CO}$ sites. Whether another CO pathway is active is not known, but the Mus81 protein clearly does not play an essential role during meiosis given the normal fertility of Mus81 mutant mice (McPherson et al. 2004, Dendouga et al. 2005). Several observations show that the repair of DSB towards $\mathrm{CO}$ or $\mathrm{NCO}$ is indeed regulated in mammals. First, the distribution of Msh4 foci, which mark most if not all DSB repair events, significantly differs from that of Mlh1, suggesting that all DSB sites do not have the same probability to become a CO. Second, when measured at the molecular level at various $\mathrm{CO}$ hotspots, variable ratios $\mathrm{CO} / \mathrm{NCO}$ were found. Specific functions are thus predicted to be required for the $\mathrm{CO} / \mathrm{NCO}$ decision, and how this regulation works remains to be understood. Interestingly, Mlh1 and 3 are specifically required for the $\mathrm{CO}$ pathway, not for NCO (Guillon et al. 2005; Svetlanov, Baudat, Cohen \& de Massy personal communication). However, unlike in S. cerevisiae, Msh4/5 functions do not seem to be exclusively involved in $\mathrm{CO}$ formation given the localization of the proteins and the mutant phenotypes. The lack of requirement for Msh4/5 for $\mathrm{NCO}$ in yeast might be related to the fact that the NCO pathway is a default pathway. Instead, in mice, Msh4/5 might also participate in NCO regulation in order to ensure the proper frequency and distribution of these events (Figure 3). This raises the question of what is the function of NCO events, which can be envisioned in at least two ways: (1) Could the process of NCO formation play a role in promoting homologous pairing, or contributing to bring homologous chromosome axis together? (2) Could the outcome of NCO confer an evolutionary advantage such as removal of DNA elements from the genome? The answers seem to differ between organisms, and it is possible that the NCO play a more critical role with respect to pairing in mammals and thus require specific functions such as Msh4/5 and possibly Sycp1. The combination of various approaches and comparisons between organisms, already extremely fruitful, is expected to continue to provide a better understanding of the regulation of DSB repair and CO control.

\section{Acknowledgements}

This work was supported by grants from Centre National de la Recherche Scientifique, Association pour la Recherche Contre le Cancer (ARC3723) and Agence Nationale de la Recherche (ANR-06-BLAN0160-01).

\section{References}

Allers T, Lichten M (2001) Intermediates of yeast meiotic recombination contain heteroduplex dna. Mol Cell 8: 225-231.

Alpi A, Pasierbek P, Gartner A, Loidl J (2003) Genetic and cytological characterization of the recombination protein RAD51 in Caenorhabditis elegans. Chromosoma 112: 6-16.

Anderson LK, Reeves A, Webb LM, Ashley T (1999) Distribution of crossing over on mouse synaptonemal complexes using immunofluorescent localization of MLH1 protein. Genetics 151: $1569-1579$.

Argueso JL, Wanat J, Gemici Z, Alani E (2004) Competing crossover pathways act during meiosis in Saccharomyces cerevisiae. Genetics 168: 1805-1816.

Ashley T, Plug AW, Xu J et al. (1995) Dynamic changes in Rad51 distribution on chromatin during meiosis in male and female vertebrates. Chromosoma 104: 19-28.

Barlow AL, Benson FE, West SC, Hulten MA (1997) Distribution of the Rad51 recombinase in human and mouse spermatocytes. EMBO J 16: 5207-5215.

Barlow AL, Hulten MA (1998) Crossing over analysis at pachytene in man. Eur J Hum Genet 6: 350-358.

Bishop DK, Zickler D (2004) Early decision; meiotic crossover interference prior to stable strand exchange and synapsis. Cell 117: 9-15.

Bocher T, Barusevicius A, Snowden T et al. (1999) hMSH5: a human MutS homologue that forms a novel heterodimer with hMSH4 and is expressed during spermatogenesis. Cancer Res 59: 816-822.

Borner GV, Kleckner N, Hunter N (2004) Crossover/noncrossover differentiation, synaptonemal complex formation, and regulatory surveillance at the leptotene/zygotene transition of meiosis. Cell 117: 29-45. 
Broman KW, Weber JL (2000) Characterization of human crossover interference. Am J Hum Genet 66: 1911-1926.

Broman KW, Murray JC, Sheffield VC, White RL, Weber JL (1998) Comprehensive human genetic maps: individual and sex-specific variation in recombination. Am J Hum Genet 63: 861-869.

Broman KW, Rowe LB, Churchill GA, Paigen K (2002) Crossover interference in the mouse. Genetics 160: 1123-1131.

Brown PW, Judis L, Chan ER et al. (2005) Meiotic synapsis proceeds from a limited number of subtelomeric sites in the human male. Am J Hum Genet 77: 556-566.

Buard J, Vergnaud G (1994) Complex recombination events at the hypermutable minisatellite CEB1 (D2S90). EMBO J 13: 3203-3210.

Buard J, Shone AC, Jeffreys AJ (2000) Meiotic recombination and flanking marker exchange at the highly unstable human minisatellite CEB1 (D2S90). Am J Hum Genet 67: 333-344.

Buard J, De Massy B (2007) Playing hide and seek with mammalian meiotic crossover hotspots. Trends Genet, Apr 12 [Epub ahead of print].

Codina-Pascual M, Campillo M, Kraus J et al. (2006) Crossover frequency and synaptonemal complex length: their variability and effects on human male meiosis. Mol Hum Reprod 12: 123-133.

Cohen PE, Pollard JW (2001) Regulation of meiotic recombination and prophase I progression in mammals. Bioessays 23: 996-1009.

Cromie GA, Hyppa RW, Taylor AF, Zakharyevich K, Hunter N, Smith GR (2006) Single Holliday junctions are intermediates of meiotic recombination. Cell 127: 1167-1178.

De Boer E, Heyting C (2006) The diverse roles of transverse filaments of synaptonemal complexes in meiosis. Chromosoma 115: $220-234$.

De Los Santos T, Hunter N, Lee C, Larkin B, Loidl J, Hollingsworth NM (2003) The mus $81 / \mathrm{mms} 4$ endonuclease acts independently of double-holliday junction resolution to promote a distinct subset of crossovers during meiosis in budding yeast. Genetics 164: 81-94.

De Massy B (2003) Distribution of meiotic recombination sites. Trends Genet 19: 514-522.

De Vries FA, De Boer E, Van Den Bosch M et al. (2005) Mouse Sycp1 functions in synaptonemal complex assembly, meiotic recombination, and XY body formation. Genes Dev 19: 1376-1389.

De Vries SS, Baart EB, Dekker M et al. (1999) Mouse MutS-like protein Msh5 is required for proper chromosome synapsis in male and female meiosis. Genes Dev 13: 523-531.

Dendouga N, Gao H, Moechars D, Janicot M, Vialard J, Mcgowan $\mathrm{CH}$ (2005) Disruption of murine Mus81 increases genomic instability and DNA damage sensitivity but does not promote tumorigenesis. Mol Cell Biol 25: 7569-7579.

Dietrich WF, Miller J, Steen R et al. (1996) A comprehensive genetic map of the mouse genome. Nature 380: 149-152.

Dumas D, Britton-Davidian J (2002) Chromosomal rearrangements and evolution of recombination: comparison of chiasma distribution patterns in standard and Robertsonian populations of the house mouse. Genetics 162: 1355-1366.

Edelmann W, Cohen PE, Kneitz B et al. (1999) Mammalian MutS homologue 5 is required for chromosome pairing in meiosis. Nat Genet 21: 123-127.

Froenicke L, Anderson LK, Wienberg J, Ashley T (2002) Male mouse recombination maps for each autosome identified by chromosome painting. Am J Hum Genet 71, 6.
Goedecke W, Eijpe M, Offenberg HH, VanAalderen M, Heyting C (1999) Mre11 and Ku70 interact in somatic cells, but are differentially expressed in early meiosis. Nat Genet 23: 194-198.

Guillon H, De Massy B (2002) An initiation site for meiotic crossing-over and gene conversion in the mouse. Nat Genet 32: 296-299.

Guillon H, Baudat F, Grey C, Liskay RM, De Massy B (2005) Crossover and noncrossover pathways in mouse meiosis. Mol Cell 20: 563-573.

Henderson KA, Keeney S (2005) Synaptonemal complex formation: where does it start? Bioessays 27: 995-998.

Her CT, Wu XL, Wan W, Doggett NA (1999) Identification and characterization of the mouse MutS homolog 5: Msh5. Mamm Genome 10: 1054-1061.

Hollingsworth NM, Brill SJ (2004) The Mus81 solution to resolution: generating meiotic crossovers without Holliday junctions. Genes Dev 18: 117-125.

Holloway K, Lawson VE, Jeffreys AJ (2006) Allelic recombination and de novo deletions in sperm in the human \{beta\}-globin gene region. Hum Mol Genet 15: 1099-1111.

Hunter N, Borts RH (1997) Mlh1 is unique among mismatch repair proteins in its ability to promote crossing-over during meiosis. Gene Dev 11: 1573-1582.

Hunter N, Kleckner N (2001) The single-end invasion: an asymmetric intermediate at the double-strand break to double-holliday junction transition of meiotic recombination. Cell 106: 59-70.

Jeffreys AJ, May CA (2004) Intense and highly localized gene conversion activity in human meiotic crossover hot spots. Nat Genet 36: 151-156.

Jeffreys AJ, Neumann R (2002) Reciprocal crossover asymmetry and meiotic drive in a human recombination hot spot. Nat Genet 31: $267-271$.

Jeffreys AJ, Neumann R (2005) Factors influencing recombination frequency and distribution in a human meiotic crossover hotspot. Hum Mol Genet 14: 2277-2287.

Jeffreys AJ, Neil DL, Neumann R (1998) Repeat instability at human minisatellites arising from meiotic recombination. EMBO J 17: 4147-4157.

Jeffreys AJ, Tamaki K, Macleod A, Monckton DG, Neil DL, Armour JA (1994) Complex gene conversion events in germline mutation at human minisatellites. Nat Genet 6: 136-145.

Jessop L, Rockmill B, Roeder GS, Lichten M (2006) Meiotic chromosome synapsis-promoting proteins antagonize the anticrossover activity of Sgs1. PLoS Genet 2.

Jones GH (1984) The control of chiasma distribution. Symp Soc Exp Biol 38: 293-320.

Kauppi L, Jeffreys AJ, Keeney S (2004) Where the crossovers are: recombination distributions in mammals. Nat Rev Genet 5: 413-424.

Keeney S (2001) Mechanism and control of meiotic recombination initiation. Curr Top Dev Biol 52: 1-53.

Kneitz B, Cohen PE, Avdievich E et al. (2000) MutS homolog 4 localization to meiotic chromosomes is required for chromosome pairing during meiosis in male and female mice. Genes Dev 14: 1085-1097.

Koehler KE, Cherry JP, Lynn A, Hunt PA, Hassold TJ (2002) Genetic control of mammalian meiotic recombination. I. Variation in exchange frequencies among males from inbred mouse strains. Genetics 162: 297-306. 
Koehler KE, Schrump SE, Cherry JP, Hassold TJ, Hunt PA (2006) Near-human aneuploidy levels in female mice with homeologous chromosomes. Curr Biol 16: R579-R580.

Kolas NK, Cohen PE (2004) Novel and diverse functions of the DNA mismatch repair family in mammalian meiosis and recombination. Cytogenet Genome Res 107: 216-231.

Kolas NK, Svetlanov A, Lenzi ML et al. (2005) Localization of MMR proteins on meiotic chromosomes in mice indicates distinct functions during prophase I. J Cell Biol, 171: 447-458.

Kong A, Gudbjartsson DF, Sainz J et al. (2002) A high-resolution recombination map of the human genome. Nat Genet 31: 241-247.

Laurie DA, Hulten MA (1985) Further studies on chiasma distribution and interference in the human male. Ann Hum Genet 49: 203-214.

Lawrie NM, Tease C, Hulten MA (1995) Chiasma frequency, distribution and interference maps of mouse autosomes. Chromosoma 104: 308-314.

Lenzi ML, Smith J, Snowden T et al. (2004) Extreme heterogeneity in the molecular events leading to the establishment of chiasmata during meiosis I in human oocytes. Am J Hum Genet 76: $112-127$.

Lynn A, Koehler KE, Judis L et al. (2002) Covariation of synaptonemal complex length and mammalian meiotic exchange rates. Science, 6: 6.

Lynn A, Ashley T, Hassold T (2004) Variation in human meiotic recombination. Annu Rev Genomics Hum Genet 5: 317-349.

Lynn A, Schrump S, Cherry J, Hassold T, Hunt P (2005) Sex, not genotype, determines recombination levels in mice. Am J Hum Genet 77: 670-675.

Mahadevaiah SK, Turner JM, Baudat F et al. (2001) Recombinational DNA double-strand breaks in mice precede synapsis. Nat Genet 27: 271-276.

Malkova A, Swanson J, German M et al. (2004) Gene conversion and crossing over along the 405-kb left arm of Saccharomyces cerevisiae chromosome VII. Genetics 168: 49-63.

Marcon E, Moens P (2003) MLH1p and MLH3p localize to precociously induced chiasmata of okadaic-acid-treated mouse spermatocytes. Genetics 165: 2283-2287.

McPherson JP, Lemmers B, Chahwan R et al. (2004) Involvement of mammalian Mus81 in genome integrity and tumor suppression. Science 304: 1822-1826.

McVean GA, Myers SR, Hunt S, Deloukas P, Bentley DR, Donnelly P (2004) The fine-scale structure of recombination rate variation in the human genome. Science 304: 581-584.

Meuwissen, RL, Meerts I, Hoovers JM, Leschot NJ, Heyting C (1997) Human synaptonemal complex protein 1 (SCP1): isolation and characterization of the cDNA and chromosomal localization of the gene. Genomics 39: 377-384.

Moens PB, Chen DJ, Shen ZY et al. (1997) Rad51 immunocytology in rat and mouse spermatocytes and oocytes. Chromosoma 106: $207-215$.

Moens PB, Kolas NK, Tarsounas M, Marcon E, Cohen PE, Spyropoulos B (2002) The time course and chromosomal localization of recombination-related proteins at meiosis in the mouse are compatible with models that can resolve the early
DNA-DNA interactions without reciprocal recombination. $J$ Cell Sci 115: 1611-1622.

Myers S, Bottolo L, Freeman C, McVean G, Donnelly P (2005) A fine-scale map of recombination rates and hotspots across the human genome. Science 310: 321-324.

Neale MJ, Pan J, Keeney S (2005) Endonucleolytic processing of covalent protein-linked DNA double-strand breaks. Nature 436: 1053-1057.

Neumann R, Jeffreys AJ (2006) Polymorphism in the activity of human crossover hotspots independent of local DNA sequence variation. Hum Mol Genet 15: 1401-1411.

Neyton S, Lespinasse F, Moens PB et al. (2004) Association between MSH4 (MutS homologue 4) and the DNA strand-exchange RAD51 and DMC1 proteins during mammalian meiosis. Mol Hum Reprod 10: 917-924.

Novak JE, Ross-Macdonald PB, Roeder GS (2001) The budding yeast Msh4 protein functions in chromosome synapsis and the regulation of crossover distribution. Genetics 158: 1013-1025.

Oliver-Bonet M, Turek PJ, Sun F, Ko E, Martin RH (2005) Temporal progression of recombination in human males. Mol Hum Reprod 11: 517-522.

Paquis-Flucklinger V, Santuccidarmanin S, Paul R, Saunieres A, Turccarel C, Desnuelle C (1997) Cloning and expression analysis of a meiosis-specific MutS homolog: the human MSH4 gene. Genomics 44: 188-194.

Petronczki M, Siomos MF, Nasmyth K (2003) Un menage a quatre: the molecular biology of chromosome segregation in meiosis. Cell 112: 423-440.

Plug AW, Xu J, Reddy G, Golub EI, Ashley T (1996) Presynaptic association of Rad51 protein with selected sites in meiotic chromatin. Proc Natl Acad Sci USA 93: 5920-5924.

Qin J, Richardson LL, Jasin M, Handel MA, Arnheim N (2004) Mouse strains with an active $\mathrm{H} 2$-Ea meiotic recombination hot spot exhibit increased levels of H2-Ea-specific DNA breaks in testicular germ cells. Mol Cell Biol 24: 1655-1666.

Rhodes M, Straw R, Fernando S et al. (1998) A high-resolution microsatellite map of the mouse genome. Genome Res, 8: 531-542

Sage J, Martin L, Cuzin F, Rassoulzadegan M (1995) cDNA sequence of the murine synaptonemal complex protein 1 (SCP1). Biochim Biophys Acta 1263: 258-260.

Santucci-Darmanin S, Walpita D, Lespinasse F, Desnuelle C, Ashley T, Paquis-Flucklinger V (2000) MSH4 acts in conjunction with MLH1 during mammalian meiosis. FASEB $J$ 14: 1539-1547.

Schmekel K, Meuwissen RL, Dietrich AJ et al. (1996) Organization of SCP1 protein molecules within synaptonemal complexes of the rat. Exp Cell Res 226: 20-30.

Shifman S, Bell JT, Copley RR et al. (2006) A high-resolution single nucleotide polymorphism genetic map of the mouse genome. PLoS Biol 4: e395.

Shinohara A, Shinohara M (2004) Roles of RecA homologues Rad51 and Dmc1 during meiotic recombination. Cytogenet Genome Res, 107: 201-207.

Shiroishi T, Koide T, Yoshino M, Sagai T, Moriwaki K (1995) Hotspots of homologous recombination in mouse meiosis. Adv Biophys 31: 119-132. 
Snowden T, Acharya S, Butz C, Berardini M, Fishel R (2004) hMSH4-hMSH5 recognizes Holliday junctions and forms a meiosis-specific sliding clamp that embraces homologous chromosomes. Mol Cell 15: 437-451.

Tarsounas M, Morita T, Pearlman RE, Moens PB (1999) RAD51 and DMC1 form mixed complexes associated with mouse meiotic chromosome cores and synaptonemal complexes. J Cell Biol, 147: 207-219.

Tease C, Hartshorne GM, Ulten MA (2002) Patterns of meiotic recombination in human fetal oocytes. Am J Hum Genet 70: 1469-1479.

Vallente RU, Cheng EY, Hassold TJ (2006) The synaptonemal complex and meiotic recombination in humans: new approaches to old questions. Chromosoma 115: 241-249.

Wang TF, Kleckner N, Hunter N (1999) Functional specificity of MutL homologs in yeast: evidence for three Mlh1-based heterocomplexes with distinct roles during meiosis in recombination and mismatch correction. Proc Natl Acad Sci USA 96: 13914-13919.

Woods LM, Hodges CA, Baart E, Baker SM, Liskay M, Hunt PA (1999) Chromosomal influence on meiotic spindle assembly: abnormal meiosis I in female Mlh1 mutant mice. J Cell Biol 145: 1395-1406.

Xu H, Beasley MD, Warren WD, Van Der Horst GT, McKay MJ (2005) Absence of mouse REC8 cohesin promotes synapsis of sister chromatids in meiosis. Dev Cell 8: 949-961.

Yauk CL, Bois PR, Jeffreys AJ (2003) High-resolution sperm typing of meiotic recombination in the mouse MHC E(beta) gene. EMBO J 22: 1389-1397.

Zenvirth D, Richler C, Bardhan A et al. (2003) Mammalian meiosis involves DNA double-strand breaks with 3' overhangs. Chromosoma 111: 369-376. 